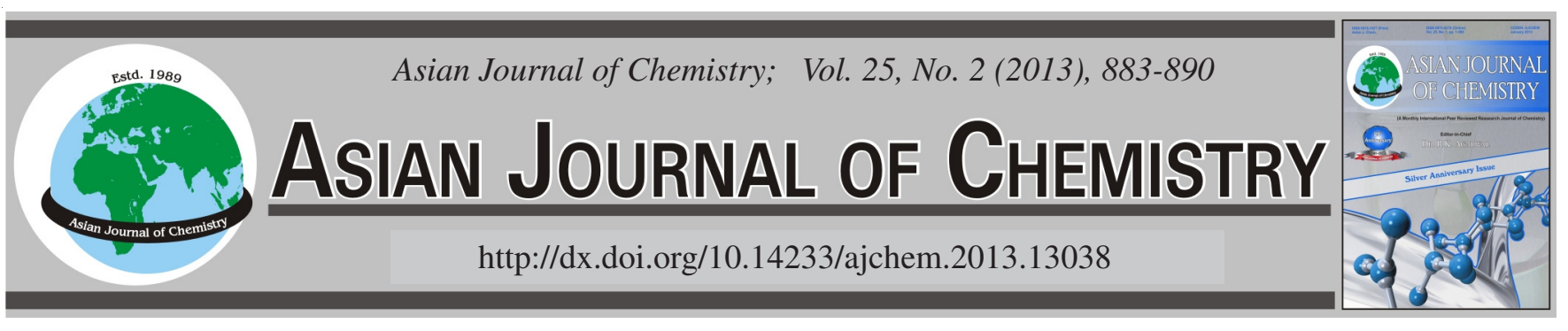

\title{
Grinding Effect of Rotation Speed on the Surface Changes of Multi-Walled Carbon Nanotubes and Their Dispersion Ability
}

\author{
B. Munkhbayar ${ }^{1}$, Munkhjargal Bat-Erdene ${ }^{2}$, Byeongdae Chol $^{2}$, \\ TAewheE Joung ${ }^{3}$, Hanshik Chung ${ }^{4}$ and Hyomin JeOng,*
}

\begin{abstract}
${ }^{1}$ Department of Energy and Mechanical Engineering, Gyeongsang National University, 445 Inpyeong Dong, Tongyeong, Gyeongsangnamdo 650-160, South Korea

${ }^{2}$ Department of Seafood Science and Technology, Gyeongsang National University, 445 Inpyeong Dong, Tongyeong, Gyeongsangnamdo 650-160, South Korea

${ }^{3}$ Eco-Friendly Heat \& Cold Energy Mechanical Research Team, Gyeongsang National University, 445 Inpyeong Dong, Tongyeong, Gyeongsangnamdo 650-160, South Korea

${ }^{4}$ Department of Energy and Mechanical Engineering, The Institute of Marine Industry, Gyeongsang National University, 445 InpyeongDong, Tongyeong, Gyeongsangnamdo 650-160, South Korea
\end{abstract}

*Corresponding author: Fax: +82 55 6403188; Tel: +82 10 95483184; E-mail: hmjeong@ gnu.ac.kr

The present work provides basic insight to largely improving the dispersion of treated multi-walled carbon nanotubes in aqueous solution with grinding and ultrasonication. The present study consists of dry and wet grinding of multi-walled carbon nanotubes with rotation speeds of 200, 300, 400 and $500 \mathrm{rpm}$ and both type of grinding with highest rotation speeds assisted to ultra-sonication dispersion of multi-walled carbon nanotubes in aqueous solutions. A simple method of purifying process also has been discussed in this study. The | results demonstrate the grinding method has urgent advantage for dispersion of multi-walled carbon nanotubes in aqueous solution: grinding can break up large multi-walled carbon nanotube aggregates into a number of small aggregates along with exfoliation to individual nanotubes which can easy the work loading of ultrasonication for dispersing multi-walled carbon nanotubes.

Key Words: Rotation speeds, Multi-walled carbon nanotubes, Nanofluids, Ultrasonication.

ᄂ - - - - - - - - - - - - - - - - - - - - - - - - -

\section{INTRODUCTION}

In the world, many published scientific papers already presented about grinding and dispersion of multi-walled carbon nanotubes in base fluids by using only ultrasonication or few variation of rotation speed in dry or wet grinding alone. The additional of this study presents a comparison of several variation of grinding speeds in dry and wet both conditions on the carbon nanotubes and their highest grinding speeds assisted to ultrasonication dispersion of carbon nanotubes in water solution. And also a simple method of purifying carbon nanotubes using nitric acid and sulfuric acid has been described.

Carbon nanotubes (CNTs) have multifunctional properties that are potentially useful in many applications due to their superior mechanical, chemical and electrical properties ${ }^{1,2}$. For this reason, many studies over the last decade have been performed with CNTs. However, there are two main issues for their applications in structural materials. First, entanglement of CNTs occurs due to their long and winding shapes, as well as due to van deer Waals forces between them. Second, weak interfacial interactions between the CNTs and the matrix occur due to the hydrophobic surfaces of the CNTs. These phenomena degrade the material properties of CNTs reinforced composites. In order to compensate for these issues, many studies have been conducted with various dispersion methods, such as ultrasonication, chemical functionalization and mechanical grinding. Due to its simplicity and ease of use, ultrasonication is generally used to disperse $\mathrm{CNTs}^{3}$. Therefore, in this study has been purposed to present these all of methods.

The grinding process is also used to disperse agglomerated CNTs. The additional of planetary ball mill was to install pots on a disk and both are rotated simultaneously and separately at high speed. Such high-speed rotation of both the pot and the disk makes the balls move strongly and violently, leading to impacts with each other of high energy which improves grinding performance. A significant grinding rate is obtained when the mill pot is rotated near the critical speed ratio counter-directionally to the disk revolution, also at near 
the critical speed ratio ${ }^{4-9}$. The configuration of the mill operation is shown in Fig. 1.

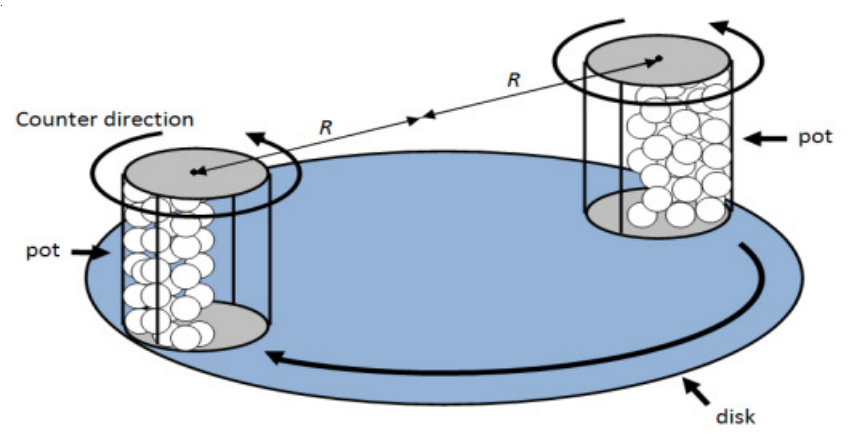

Fig. 1. Configuration of the planetary ball mill

Carbon nanotubes have a wide range of applications including energy storage, biosensors, composites, field emission devices, electronic components and probe tips, etc. Carbon nanotubes have an extraordinary structure and more importantly, possess attractive properties and promising application perspectives ${ }^{10}$. However, pristine CNTs contain a large amount of impurities, such as catalyst particles, amorphous carbon and multishell carbon nanocapsules. These impurities are a serious impediment to characterize the properties of CNTs extensively and further explore the application of CNTs. A large variety of purifying methods, such as physical separation, gas-phase oxidation, liquid-phase oxidation and combinatorial purification have been emerged to purify the CNTs.

Physical separation is only useful for the preparation of small amounts of purified samples. Gas-phase oxidation is little efficient for the removal of graphitic impurities and catalyst particles. Liquid-phase oxidation is generally carried out with acid solutions such as $\mathrm{HNO}_{3}$ or mixtures of $\mathrm{H}_{2} \mathrm{SO}_{4} / \mathrm{HNO}_{3}$ or $\mathrm{H}_{2} \mathrm{SO}_{4} / \mathrm{KMnO}_{4}$. In additional of liquid-phase oxidation method has been purified efficient for eliminating impurities ${ }^{11,12}$.

A high resolution imaging was done with a scanning electron microscope (SEM) imaging was done to ascertain the structure of nanoparticles in the fluid. Particularly, carbon nanotubes are bundled with strong van deer Waals high interaction energy of $c a .500 \mathrm{eV} / \mu \mathrm{m}$ of tube-tube contact ${ }^{13}$. Measurement of the length and purity of MWCNTs has been used transmission electron microscopy (TEM). The particle sizing analysis (Mastersizer 2000, Malvern Instruments) has been used to measure main particle size of ground MWCNTs. Mastersizer 2000 particle size analyzer is for measurement of emulsions, suspensions and dry powders range of from 0.01 $\mu \mathrm{m}$ to $2000 \mu \mathrm{m}$. The dispersion quality of MWCNTs was confirmed by UV-VIS (UV-1800, Shimadzu) spectrophotometer. UV-VIS spectrophotometer measurements are used to quantitatively characterize colloidal stability of the dispersions as a new method $^{14}$.

Li et al. ${ }^{15}$ studied that the carbon nanotubes were treated by high-energy ball milling processing for different milling times and the samples were observed by high resolution transmission electron microscopy (HRTEM). Ahn et al. ${ }^{16}$ described that the multi-walled carbon nanotubes tend to be compacted by impact of the balls and form agglomerates with increased size during the ball milling process as observed by scanning electron microscopy. The compactness or strength of CNT agglomerates influenced their dispersability. Ruban et al. ${ }^{17}$ reported the one step water assisted synthesis of high quality carbon nanotubes directly from graphite and the carbon nanotubes was incorporated with zinc metal.

The main objective of this study were confronts a comparative on the dry and wet both conditions grinding with four rotation speeds i.e., 200, 300, 400 and $500 \mathrm{rpm}$, their highest grinding speeds assisted to ultrasonication dispersion of pristine and purified MWCNTs in aqueous solutions. In this study were grinding, purifying and ultrasonication process has been investigated to cutting length of CNTs and decreasing size, improving CNTs quality and spreading particles in to water, respectively.

\section{EXPERIMENTAL}

The materials used in this study were multiwalled carbon nanotubes synthesized by catalytic chemical vapour deposition (Carbon Nanomaterial Technology Co., Ltd, South Korea). The diameter and length range of the MWCNTs was $c a .20 \mathrm{~nm}$ and $c a .5 \mu \mathrm{m}$, respectively and the purity was greater than $95 \%$, with less than $3 \%$ amorphous carbon. Grinding devices used in this work were planetary ball mills made by Haji Engineering, Korea. The collision medium was zirconia $\left(\mathrm{ZrO}_{2}\right)$ balls (Haji Engineering, Korea) with a diameter of $3 \mathrm{~mm}$ monosized spheres. The sulphuric and nitric acid (provided by Matsunoen Chemicals Co., Ltd, Japan) in concentrations of $c a .98 \%$ and $c a .63 \%$, respectively, were used for purifying process. The ethyl alcohol (provided by Duksan Pure Chemicals, Korea) used for the acid neutralizing. All dispersion experiments were carried out with distilled water. The model of ultrasonication was Branson ultrasonication 1510E-DTH (Branson Ultrasonic Corporation 41, Danbury, CT 06813, USA). Calorimetric was performed to measure the output power and frequency of ultrasound, which was $63 \mathrm{~W}$ and 42 KHz. The properties of MWCNTs present simulation work are tabulated in Table-1.

\begin{tabular}{lc}
\multicolumn{2}{c}{ TABLE-1 } \\
PROPERTIES OF MWCNTs \\
\hline \multicolumn{1}{c}{ Properties } & Value \\
\hline Diameter $(\mathrm{nm})$ & $\approx 20$ \\
Length $(\mu \mathrm{m})$ & $\approx 5$ \\
Purity $(\mathrm{wt} \%)$ & $>95$ \\
Thermal conductivity $\left(\mathrm{W} \mathrm{m}^{-1} \mathrm{~K}^{-1}\right)$ & Max. 3000 \\
Density $\left(\mathrm{g} / \mathrm{cm}^{3}\right)$ & 1.8 \\
\hline
\end{tabular}

Grinding process: Grinding was performed as follows: MWCNTs and zirconia balls were put into cylindrical stainless zirconia pot (40 $\mathrm{mm}$ in inner diameter and $45 \mathrm{~mm}$ in height) box. This mill had a $\mathrm{ZrO}_{2}$ lining with $56 \mathrm{~cm}^{3}$ volume of milling 2 pots which was filled with about $80 \mathrm{vol}$. $\%$ of $3 \mathrm{~mm}$ spherical $\mathrm{ZrO}_{2}$ balls and MWCNTs. In the experiment of dry grinding $0.1 \mathrm{~g}$ MWCNTs were placed in each pot and 1400 stainless zirconia balls ( $120 \mathrm{~g}$ balls) added in to each pot. In the experiment of wet condition was $20 \mathrm{~mL}$ distilled water added in to each pot. The pot rotation was set counter to the disk revolution. In order to compare the effective processing parameters, grinding of the MWCNT particles was performed at various 
grinding speeds and times in dry and wet (using distilled water) conditions. Specifically, the agitator-applied grinding speeds were 200 revolutions per min (rpm), 300, 400 and $500 \mathrm{rpm}$ and the grinding time was $1 \mathrm{~h}$ for all grinding experiment. In this grinding experiment, changes in the particle sizes and shapes of the ground powders with increasing grinding speeds were analyzed by particle size analysis (PSA) (Mastersizer 2000, Malvern Instruments) and a scanning electron microscope (SEM) respectively. SEM results are in agreement with the PSA measurements.

MWCNTs-purifying process: At present, the purest CNTs $(>95 \%)$ with amorphous carbon less than $3 \%$ are prepared by their CVD growth in the presence of catalysts on special supports. In this case, the amount of amorphous carbon is relatively small, graphite is virtually absent and catalyst metal particles can be eliminated by comparatively brief treatment with an $\operatorname{acid}^{18}$. A simple method for purifying multi-walled carbon nanotubes using nitric acid and sulfuric acid is described. The pristine MWCNTs were dispersed in $0.5 \%$ (v/ v) nitric acid and sulphuric acid concentrate $\left(\mathrm{HNO}_{3} / \mathrm{H}_{2} \mathrm{SO}_{4}=\right.$ $1 / 3, \mathrm{v} / \mathrm{v}$ ) by performing ultrasonication for $5 \mathrm{~h}$ to remove the impurities and add exterior activeness, then washed by anhydrous ethanol and deionized water. Otherwise, the chemical reformation process was also employed where chemically treated hydroxyl radicals adhered to the MWCNTs surface. The acidic mixture of MWCNTs containing carboxyl radicals was then diluted by adding deionized water. This process of washing and filtration processes was repeated until a $\mathrm{pH}$ of 7 was achieved, which was then filtered with a vacuum filter. The moisture was removed by placing the MWCNTs mixture in a vacuum oven for $12 \mathrm{~h}$ maintaining at $100^{\circ} \mathrm{C}$, to give MWCNTs functionalized with carboxylic acid (MWCNTs$\mathrm{COOH}$ ). Fig. 2 showing the schematic illustration of the purifying of MWCNTs using by two kinds of acid and ultrasonication. Pristine and purified MWCNTs were evaluated by TEM and SEM.

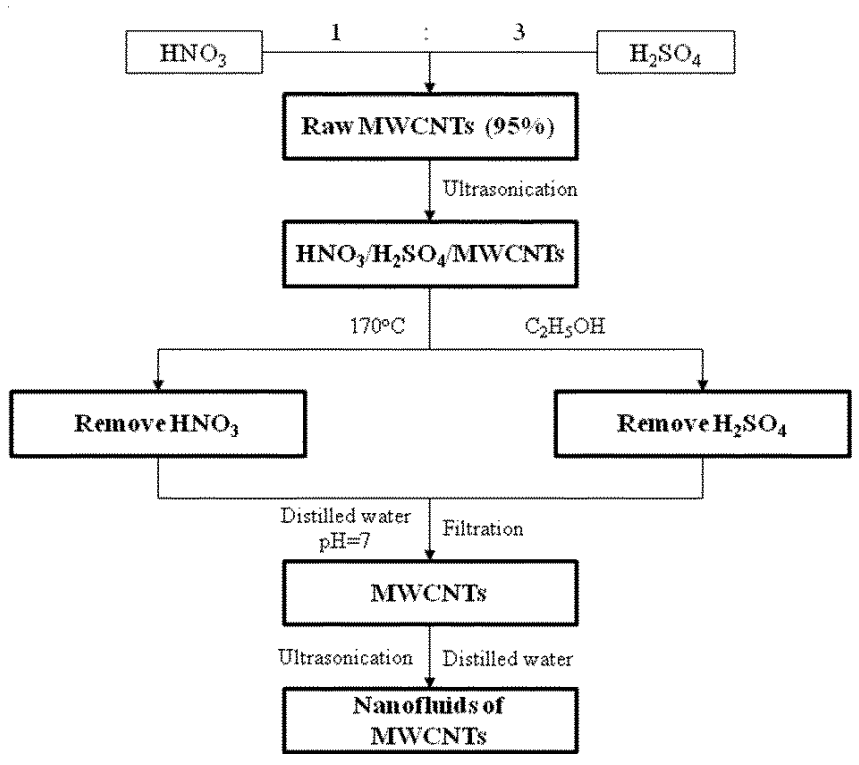

Fig. 2. Schematic illustration of the purifying MWCNTs
Dispersion process of MWCNTs: In order to compare the dispersion quality of pristine, purified, dry and wet ground with $500 \mathrm{rpm}$ rotation speed of MWCNTs in aqueous solutions were confirmed. The concentration of these samples of MWCNTs in water (0.01 wt \%), the carbon nanotubes were dispersed in a concentration of $0.1 \mathrm{~g} / \mathrm{l}$ in an aqueous solution. These all samples were ultrasonicated for $1 \mathrm{~h}$ in order to spread MWCNTs in aqueous solution. Dispersions of MWCNTs were monitored by transmission electron microscopy (TEM) and UV-visible spectrophotometer (UV-1800, Shimadzu, Japan) operating between the wavelengths of 200-1100 nm.

\section{RESULTS AND DISCUSSION}

Fig. 3 shows the diameter and length range of the pristine MWCNTs was $c a .20 \mathrm{~nm}$ and $c a .5 \mu \mathrm{m}$, respectively and the purity was greater than $95 \%$ with less than $3 \%$ of amorphous carbon. in high magnification of SEM micrographs. High magnification reveals clearly the individual CNTs and some of their characteristic features (size, shape, purity). This present study were focus on the comparative study on the dry and wet grinding at several variations of rotation speeds at 200, 300, 400 and $500 \mathrm{rpm}$, their highest grinding speed assisted to ultrasonication dispersion of multi-walled carbon nanotubes (MWCNTs). The result has been monitored by scanning electron microscopy (SEM), transmission electron microscopy (TEM), particle sizing analysis (PSA) and a UV-visible spectrophotometer.
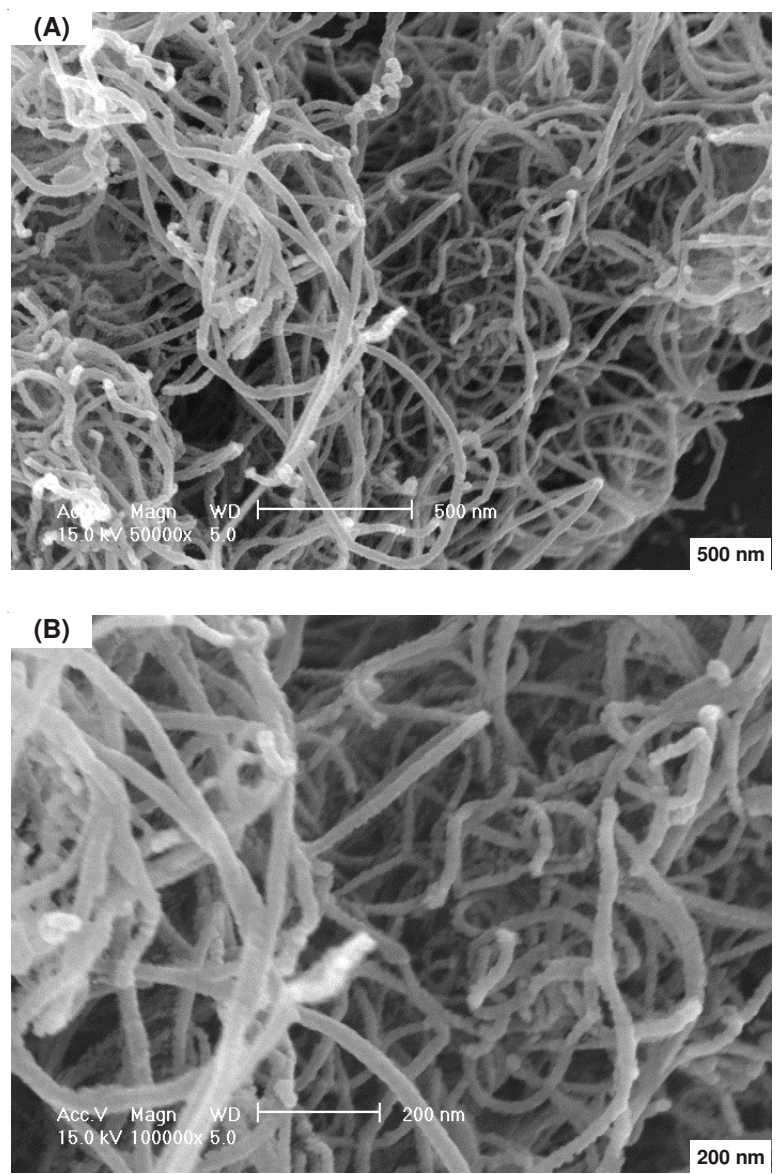

Fig. 3. SEM micrographs showing the shape of the MWCNTs employed in the current study (A) low resolution (B) high resolution 
Grinding of CNTs: In order to compare the grinding efficiency between the changes of rotation speeds in dry and wet grinding, the following experiment was performed. Four batches of MWCNTs powders were ground at rotation speeds of 200, 300, 400 and $500 \mathrm{rpm}$ in the dry and wet condition. Changes in the particle sizes and shapes of the ground powders with increasing grinding speeds were analyzed using a particle size analyzer and a scanning electron microscope (SEM). Fig. 4 presents high magnification SEM micrographs of the distinction between the wet and dry grinding of MWCNTs at rotation speeds of 200, 300, 400 and $500 \mathrm{rpm}$. Fig. 4(a) shows the dry grinding and Fig. 4(b) shows the wet grinding of CNTs.
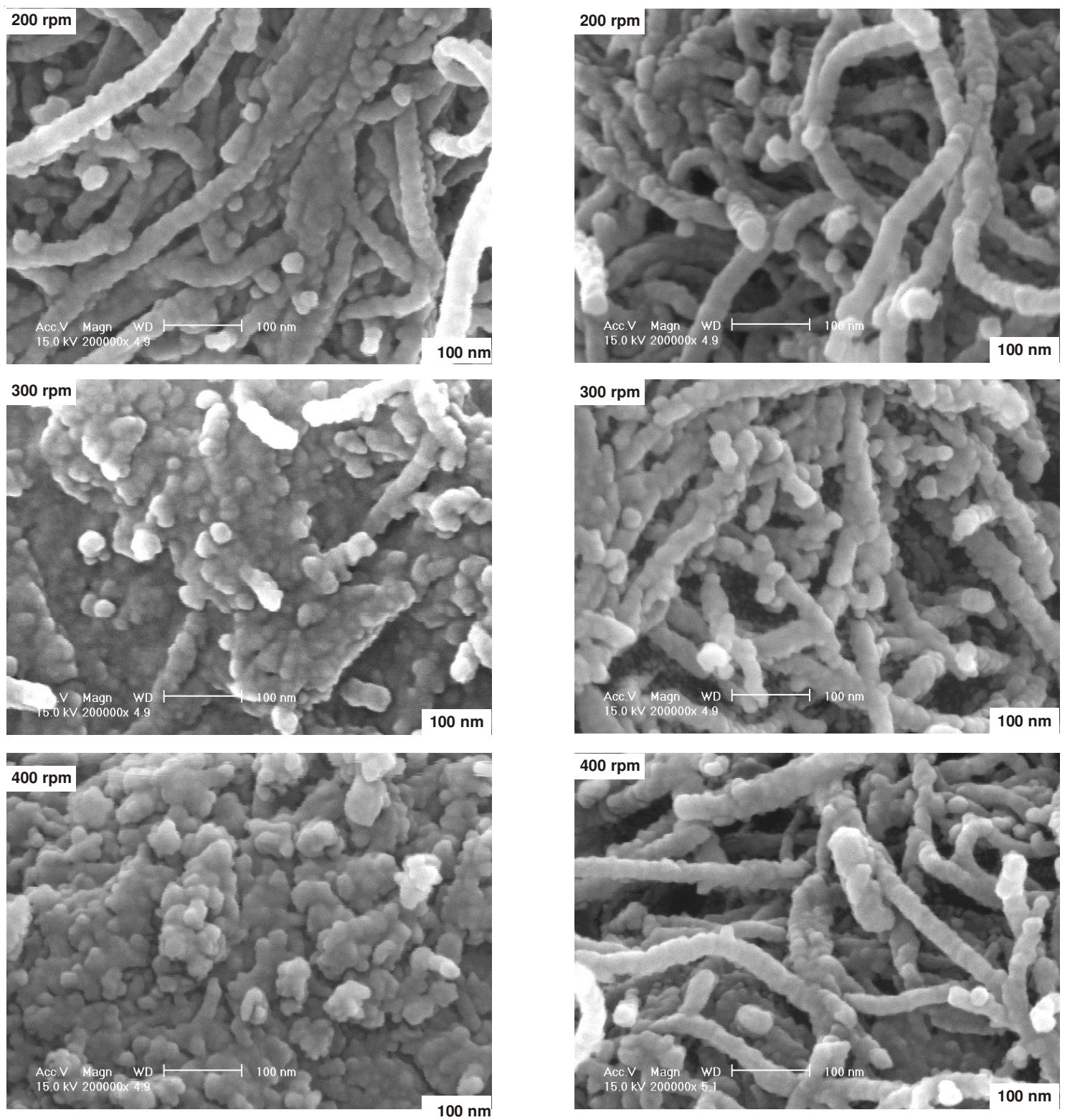

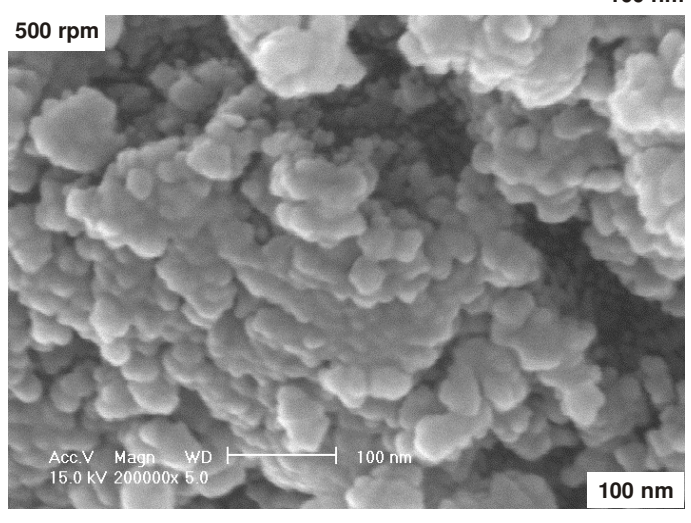

(a)

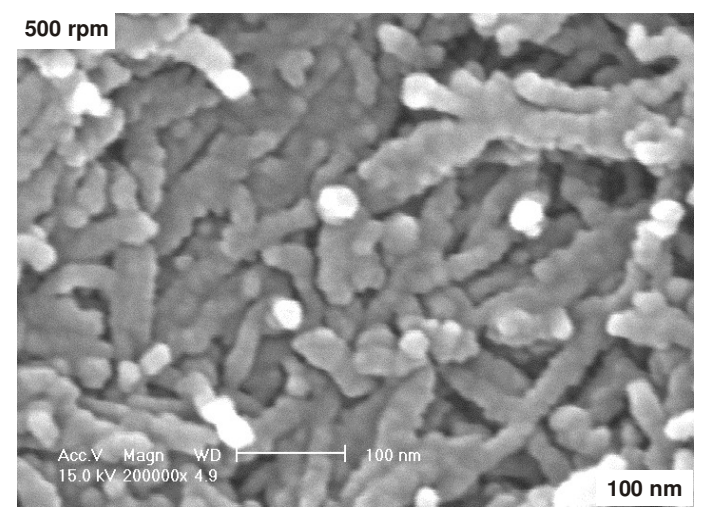

(b)

Fig. 4. High magnification of SEM micrographs showing (a) dry and (b) wet grinding of MWCNTs at different rotation speeds 
In general, once powders are grinding process resulting in the agglomeration of the particles with increasing grinding time and rotation speed. Particle break up happens from the relative impact velocity of balls colliding with each other or against the mill pot wall during the grinding. The SEM micrographs were also utilized to characterize the morphology, uniformity and thickness of the CNTs. In Fig. 4(a), the CNTs particles had started to crush by plastic deformation under the impact of the balls, which were forming particles having rough surfaces in $200 \mathrm{rpm}$. The rotation speed of $200 \mathrm{rpm}$ in dry condition can be seen that MWCNTs powders became irregular in shape and going to be agglomerated. When the grinding speed was increased, crushing of the particles were noticeable as seen in the SEM micrographs for the grinding speeds of 300 to $500 \mathrm{rpm}$ in dry conditions. The particle crushing was noticeable as seen in that SEM micrograph at rotation speed of $300 \mathrm{rpm}$ of dry grinding. The MWCNTs particles became flattened, broken and started to agglomerate from rotation speed of $300 \mathrm{rpm}$ in dry grinding. Further increasing the grinding speed of $500 \mathrm{rpm}$ can be seen MWCNTs powders thoroughness crushed, formed particles have rough surfaces and their shapes became rugged, flattened and shortened. But MWCNTs particles were noticeable agglomerated at rotation speed of $500 \mathrm{rpm}$ in dry condition. And also the grinding speed of $500 \mathrm{rpm}$ showing, the more particles are crushed the more agglomerations are appearances. Firstly, ungrounded and grounded MWCNTs at a rotation speed of $200 \mathrm{rpm}$ results were observed their length were longer than $200 \mathrm{~nm}$. However, ground MWCNTs at rotation speeds from 300 to $500 \mathrm{rpm}$ in dry grinding results was observed their length became shorter than $100 \mathrm{~nm}$.

Contrary to the case of the dry grinding, little bit different grinding behaviour was observed in case of the wet grinding MWCNTs powders as shown in the series of SEM micrographs in Fig. 4(b). In Fig. 4(b), the rotation speed of $200 \mathrm{rpm}$ in wet and dry conditions seem to produce almost the same results, there had not complete change about particle breakage. The beginning point of particles breakage in the wet grinding was at rotation speeds of 300 and $400 \mathrm{rpm}$. The ball milling speed increased to $500 \mathrm{rpm}$, it can be seen particles became broken and agglomerated in the SEM micrograph. The structures of the MWCNTs seem to randomness and granular structures at rotation speed of $500 \mathrm{rpm}$. MWCNTs are aggregated into ropes or knotted lumps due to strong inter-tube van deer Waals attraction and some of them demonstrate a few local kinks and bends. During the grinding process, the powder mixture is subjected to the high inter-particle and balls collision in a high-speed rotation speeds. In addition, improvement in the specific surface area with increasing grinding speed was observed. The grinding of CNTs resulted in shortened and densely packed CNTs with increased grinding speed due to increased cleavage.

Fig. 4 shows particles breakage in grinding of dry conditions higher than comparing with wet conditions. In case of dry conditions were only particles directly filled in pots where as in case of wet conditions were particles mixing with water filled in pots. Accordingly the wet conditions of CNTs still have long fiber shapes. During the grinding process, impact forces of balls are different in dry and wet conditions. The impact forces of balls are lower in the wet condition grinding.

The reason of SEM results, ground MWCNTs of dry conditions samples selected for next step of measuring device. While these SEM observations give qualitative information as to the changes in the shapes and sizes of the particles during the grinding, quantitative information on the grinding behaviour can be obtained from the PSA. The ground MWCNTs at rotation speeds of 200, 300, 400 and $500 \mathrm{rpm}$ in dry conditions samples were characterized by particle size analysis. This method demonstrates the important application of carbon nanotubes in the field of particle sizing analysis. The principle of particle size measurement of this experiment (Mastersizer 2000) is the laser scattering and diffraction method. This method is widely used in the powder technology fields. Especially it is used when lots of investigators measure CNT powder. The effect of particle fraction of various grinding speed on the particle size response was investigated from particle size 0.01 to $1000 \mu \mathrm{m}$ (Fig. 5). It can be seen in Fig. 5 increasing the grinding speeds from 200 to $400 \mathrm{rpm}$ resulted peaks of particle sizes were still to down, as the rotation speed of $500 \mathrm{rpm}$ peak of particle sizes were increased back. The mean particles sizes of CNTs at rotation speed of 200, 300, 400 and $500 \mathrm{rpm}$ in dry conditions were $30.2,19.95,14.15$ and 34.67 $\mu \mathrm{m}$, respectively. The CNTs at a rotation speed of $200 \mathrm{rpm}$ result showed particle size analyzer peak to larger particle size regions. In the grinding speeds of 400 and $500 \mathrm{rpm}$ results showed peak positions to smaller particle size regions. The primary CNT agglomerate size distributions are given in Fig. 6 as accumulative curves. The smallest agglomerates were found for ground with highest rotation speed of CNTs. All detected particles sizes were between the ranges from 0.5 to $100 \mu \mathrm{m}$. However $10 \%$ of the 400 and $500 \mathrm{rpm}$ for ground powder contained agglomerates smaller than the measurement of 4 $\mu \mathrm{m}$. And also $10 \%$ of $300 \mathrm{rpm}$ and $10 \%$ of the $200 \mathrm{rpm}$ results were about 5 and $9 \mu \mathrm{m}$, respectively. But from the 50 to $90 \%$ of all ground MWCNTs were noticeable observed in particles sizes. Significantly higher agglomerates sizes were measurement range of about 45 to $90 \mu \mathrm{m}$ observed in ground of $200 \mathrm{rpm}$. The smallest agglomerates were measurement

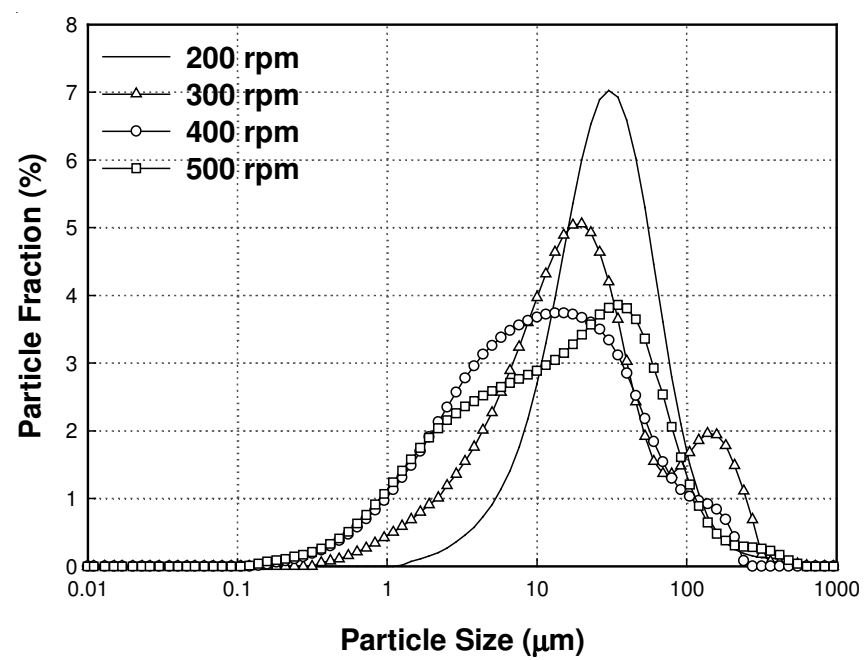

Fig. 5. Influence of particle size on the particle fraction response for grinded sample of four kind of rotation speed 


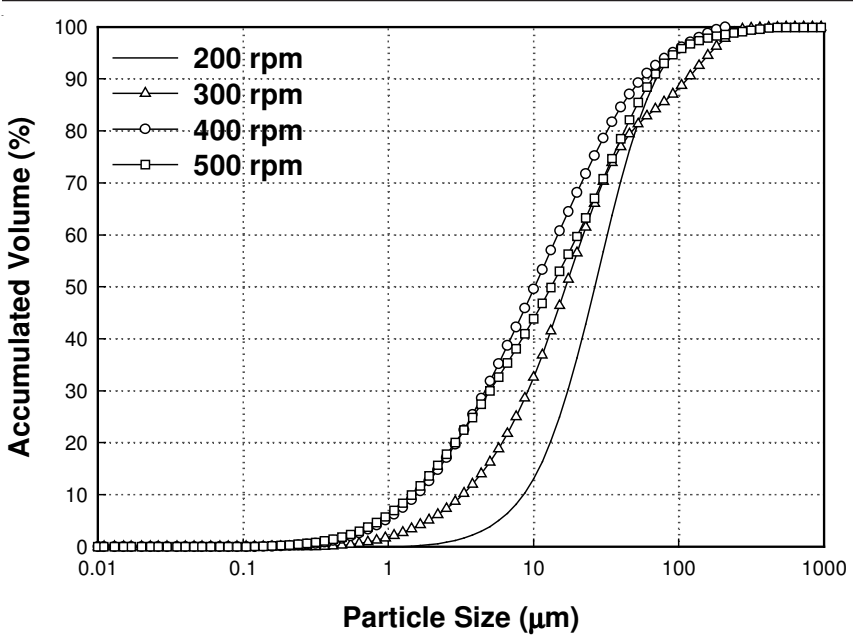

Fig. 6. Influence of particle size on the accumulation volume response for grinded sample of four kind of rotation speed

range of about 10 to $80 \mu \mathrm{m}$ observed in ground of $400 \mathrm{rpm}$. The grinding speeds of 300 and $500 \mathrm{rpm}$ particles sizes were almost similar in accumulated volume of 50 to $90 \%$. In Table-2 evident the mean particle sizes of CNTs increase with grinding speed.

\section{TABLE-2}

CHANGES OF MEAN PARTICLE SIZES OF THE GROUND MWCNTs WITH INCREASING GRINDING SPEEDS

\begin{tabular}{ccccc}
\hline Grinding speed $(\mathrm{ppm})$ & 200 & 300 & 400 & 500 \\
\hline Particle size $(\mu \mathrm{m})$ & 30.2 & 19.95 & 14.15 & 34.67
\end{tabular}

In all results showing the particles size was still decreasing and particles crushing were increasing in the grinding speeds of 200, 300 and $400 \mathrm{rpm}$. But the grinding speed of $500 \mathrm{rpm}$ result noticeable as seen particles breaks were very high and particles size was increased in that SEM micrograph and particle size analysis. The reason of this increase in particles size is because at high rotation speed the particles had broken into very small size as compare to low rotation speed. Due to which the particles had agglomerated and hence shown bigger in size. Such changes in the frequency particle size with increasing grinding speed is the indication that the powders grew in size during the grinding by coalescing with the smaller powders, i.e. agglomeration proceeded with increasing grinding speed. The grinding can break up large MWCNT aggregates into a number of small aggregates along with exfoliation to individual nanotubes. The long length of MWCNTs shortened by grinding process is much important for dispersion characteristic. Because that aggregated MWCNTs can be prevented by chemical functionalization and ultrasonication machine. In the grinding results, the SEM results are in agreement with the particle size analysis measurements.

Purification of MWCNTs: It is noted that the untreated pristine MWCNTs are generally floated and difficult to spread over on the water, which could be due to the large aspect ratio of MWCNTs and surface tension of water supporting the light MWCNTs, despite the density of the MWCNTs (1.8 g/ $\mathrm{cm}^{3}$ ) being slightly larger than that of water. For this reason, a simple method for purifying multiwalled carbon nanotubes (MWCNTs) by using nitric acid and sulfuric acid is described in this experiment. The high resolution TEM image shows that pristine MWCNTs contain catalyst and carbon particles (black arrows in Fig. 7a). The pristine CNTs contain a large amount of impurities, such as catalyst particles, amorphous carbon and multishell carbon nanocapsules. The catalyst particles are evidently embedded in the tips or the tube cores of MWCNTs. The outer walls of pristine MWCNTs are coated with a layer of amorphous carbon, which can be clearly distinguished shown in Fig. 7a. The purified MWCNTs are clean and there are no carbon particles shows in Fig. 7b. Importantly, the purified CNTs were described to their structure, amorphous carbon and carbon particles were almost completely eliminated after purification and the tips of the purified MWCNTs were opened (white arrows in Fig. 7b). Consequently, those impurities of catalyst particles, amorphous carbon and carbon particles in pristine MWCNTs were eliminated by treat with sulfuric acid and nitric acid. The crystal structure of MWCNTs body was unchanged after purifica-tion. More importantly, a certain number of carboxyl groups were formed on MWCNTs.
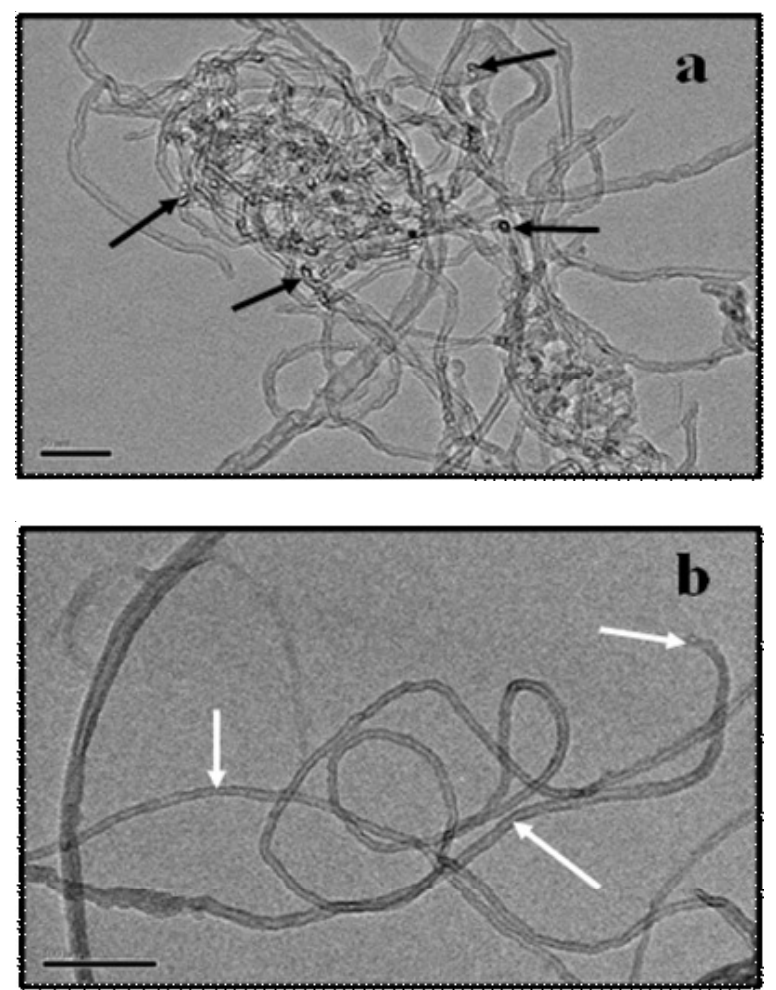

Fig. 7. High magnification of TEM micrographs showing (a) pristine MWCNTs and (b) purified MWCNTs by acids

Dispersion of MWCNTs: In order to perform the best dispersion of MWCNTs, the most important object has shape, size, structure and purity. In this dispersion study, grinding process has been investigated to decreasing particle size and controlling particle shape, the purifying experiments were performed for increasing purity of MWCNTs and the ultrasonication was used for spreading particles in to base fluid. To compare the dispersing quality of the pristine, purified, dry and wet ground with rotation speed of $500 \mathrm{rpm}$ assisted to ultrasonication dispersion of MWCNTs in aqueous solution. The dispersions were then tranquilized at room temperature 
and checked with optical microscopy. The dispersion states of pristine, purified, dry and wet ground with rotation speed of $500 \mathrm{rpm}$ assisted to dispersion of MWCNTs in aqueous solution $(0.1 \mathrm{~g} / \mathrm{L})$ by using ultrasonication for $1 \mathrm{~h}$ and their stabilities were observed after 30 days shown in Fig. 8. Fig. 8(1) shows the pristine MWCNTs were dispersed in aqueous solution by using ultrasonication for $1 \mathrm{~h}$. The dispersion states of pristine MWCNTs results were observed large amounts of pristine MWCNT aggregates on the surface of water and bottom side of bottle instead of dispersing. Fig. 8(2) shows the dispersion of purified MWCNTs were observed no agglomeration and CNTs particles probable dispersed in water, even that dispersion states were not so dark comparing with Fig. 8(3,4). The state of dry ground with rotation speed of $500 \mathrm{rpm}$ assisted to ultrasonication dispersion of MWCNTs in aqueous solution is showing the Fig. 8(3). Best dispersion state was observed that wet ground with rotation speed of $500 \mathrm{rpm}$ assisted to ultrasonication dispersion of MWCNTs in aqueous solution is showing the Fig. 8(4). The colour of the suspension treated by grinding with rotation speed of $500 \mathrm{rpm}$ and ultrasonication supported dispersion of CNTs becomes darker than that other dispersion of CNTs, indicating that wet-grinding can improve the ultrasonic dispersion efficiency of MWCNTs in water. The optical microscopy results shows the ground with rotation speed of $500 \mathrm{rpm}$ assisted to ultrasonication dispersion of MWCNTs were much better than dispersion of pristine, only purified and dry ground assisted MWCNTs.

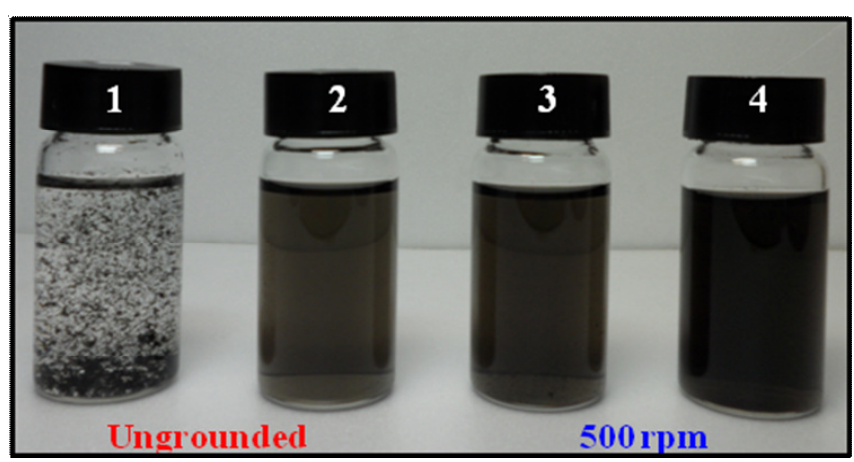

Fig. 8. Dispersion states of (1) pristine, (2) purified, (3) dry ground (4) wet ground with rotation speed of $500 \mathrm{rpm}$ assisted to ultrasonication dispersion of MWCNTs in aqueous solutions

Individual CNTs are active in the UV-VIS region and exhibit characteristic bands corresponding to additional absorption due to $1 \mathrm{D}$ van Hove singularities. Bundled CNTs, however, are hardly active in the wavelength region between 200 and $1200 \mathrm{~nm}$ their photoluminescence is quenched most probably because of carrier are tunneling between the nanotubes. Therefore, it is possible to establish a relationship between the amounts of CNTs individually dispersed in solution and the intensity of the corresponding absorption spectrum. Moreover, UV-VIS spectroscopy can be used to monitor the dynamics of this dispersion process of $\mathrm{CNTs}^{19}$.

Generally, higher absorbance of CNTs indicates better dispersion and solubility in solution. In order to compare absorption between the dispersion of the pristine, purified, dry and wet ground with rotation speed of $500 \mathrm{rpm}$ assisted MWCNTs were dispersed in aqueous solutions were charac- terized by using UV-VIS spectrophotometer (Shimadzu UV1800) operating at wavelength range of 200 to $1100 \mathrm{~nm}$. All the MWCNTs suspensions were further diluted with $0.01 \%$ (w/w) aqueous solution to a suspension of MWCNT concentration of $0.1 \mathrm{~g} / \mathrm{L}$ for test. The comparison on the absorbance of various conditions of dispersion of MWCNTs in aqueous solutions at wavelength of 200 to $1100 \mathrm{~nm}$ depicts in Fig. 9. The absorption peak at about $260 \mathrm{~nm}$ in Fig. 9 is attributed to the characteristic absorption of MWCNTs ${ }^{19}$. The low absorbance (0.518) in pristine MWCNTs prepared by ultrasonication corresponds to the poor dispersion of MWCNTs as observed in Fig. 8(1). The absorbance of dispersion of purified MWCNTs was 1.080 at a wavelength about $260 \mathrm{~nm}$. After grinding, the absorbance of MWCNTs suspension largely increases. High absorbance (1.565) showed in dry ground with rotation speed of $500 \mathrm{rpm}$ assisted to ultra-sonication dispersion of MWCNTs in aqueous solution. The maximum absorbance (about 2.36) of suspension is observed in wet ground with rotation speed of $500 \mathrm{rpm}$ assisted result. The absorb-ance of MWCNTs suspensions prepared by which method does not depend on the MWCNT concentration in a linear fashion, which is attributed to the relatively poor dispersion of MWCNTs. This indicates that the best dispersion quality of MWCNTs in suspension can be obtained by combining both the grinding and ultrasonication among these methods.

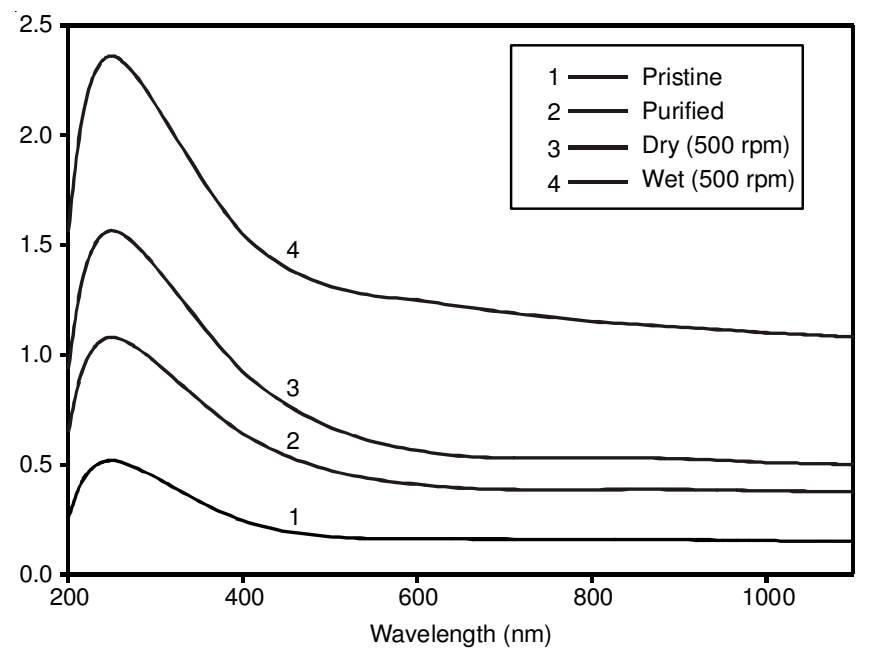

Fig. 9. UV-spectra absorbance of pristine, purified, dry and wet ground with rotation speed of $500 \mathrm{rpm}$ assisted to ultrasonication dispersion of MWCNTs in aqueous solutions at wavelength range of 200 to $1100 \mathrm{~nm}$

Finally, grinding method has urgent advantage for dispersion of MWCNTs in aqueous solution: grinding can break up large MWCNT aggregates into a number of small aggregates along with exfoliation to individual nanotubes which can easy the work loading of ultrasonication for dispersing MWCNTs. The resulting MWCNTs were clean-cut and well-separated by these methods. The grinding with wet condition process is a powerful method to decrease particle size and agglomeration of MWCNTs. In addition, a higher milling speed plays a more important role in the size reduction and dispersion of MWCNTs. This finding corroborates the results of previous studies illustrating the length reduction and distribution of MWCNTs. The 
dispersion quality of MWCNTs in aqueous solution prepared by rotation speed of $500 \mathrm{rpm}$ in wet-grinding assisted to ultrasonication is much better than the other conditions.

\section{Conclusion}

Purified MWCNTs were described to their structure, amorphous carbon and carbon particles were almost completely eliminated after purification and tips of the purified MWCNTs were opened. The crystal structure of MWCNTs shape was unchanged after purification.

The MWCNTs powders were thoroughness broken, formed particles have rough surfaces and their shapes became rugged, flattened and lengths were shortened in high grinding speed. That long length of MWCNTs shortened by grinding is very important for dispersion characteristic. That aggregated MWCNTs can be prevented by chemical surfactants and ultrasonication machine. Here, grinding method has urgent advantage for dispersion of MWCNTs in aqueous solution: grinding can break up large MWCNT aggregates into a number of small aggregates along with exfoliation to individual nanotubes which can easy the work loading of ultrasonication for dispersing MWCNTs. The dispersion quality of MWCNT is absolutely increased with high grinding speed, introduced chemical surfactants and ultrasonication.

This study just provides basic insight to largely improving the dispersion of MWCNTs in aqueous solution with grinding and ultrasonication. The dispersion quality of MWCNTs in aqueous with surfactant solution prepared by at a rotation speed of $500 \mathrm{rpm}$ in wet-grinding assisted to ultra-sonication method is far better than the other conditions.

\section{ACKNOWLEDGEMENTS}

This research was supported by Basic Science Program through the National Research Foundation of Korea (NRF) funded by the Ministry of Education, Science and Technology (No. 2012-0004544).

\section{REFERENCES}

1. S.K. Das, S.U. Choi, W. Yu and T. Pradeep, Nanofluids: Science and Technology, Wiley-Interscience, pp. 7-31, 337-348 (2007).

2. S.K. Swain and I. Jena, Asian. J. Chem., 22, 1 (2010).

3. J.H. Lee, K.Y. Rhee and S.J. Park, Int. J. Hydrogen Energ., 35, 7850 (2010).

4. H. Mio, J. Kano, F. Saito and K. Kaneko, J. Miner. Proc., 74S, S85 (2004).

5. R.M. Davis, B. McDermott and C.C. Koch, J. Metal. Mater. Trans. A, 19, 2867 (1988).

6. S. Kim, H. Chung and H. Choi, J. Am. Ceram. Soc., 95, 531 (2009).

7. J. Kano, S. Saeki, F. Saito, M. Tanjo and S. Yamazaki, J. Miner. Proc., 60, 91 (2000).

8. J. Kano and F. Saito, Powder Technol., 98, 166 (1998).

9. H. Mio, J. Kano, F. Saito and K. Kaneko, Mater. Sci. Eng. A- Struct., 332, 75 (2002).

10. K. Chu, H. Guo, C.C. Jia, F.Z. Yin, X.M. Zhang, X.B. Liang and H. Chen, Nanoscale Res. Lett., 5, 868 (2010).

11. J.-M. Yuan, X.-H. Chen, X.-H. Chen, Z.-F. Fan, X.-G. Yang and Z.-H. Chen, Carbon, 46, 1266 (2008).

12. V. Djordjevica, J. Djustebeka, J. Cveticanina, S. Velicknovica, M. Veljkovica, M. Bokorovb, B. Babic Stojica and O. Neskovica, J. Optoelectron. Adv. Mater., 8, 1631 (2006).

13. R. Rastogi, R. Kaushal, S.K. Tripathi, A.L. Sharma, I. Kaur and L.M. Bharadwaj, J. Colloid Interface Sci., 328, 421 (2008).

14. L.Q. Jiang, L. Gao and J. Sun, J. Colloid Interface Sci., 260, 89 (2003).

15. Y.B. Li, B.Q. Wei, J. Liang, Q. Yu and D.H. Wu, Carbon, 37, 493 (1999).

16. J.H. Ahn, H.S. Shin, Y.J. Kim and H. Chung, J. Alloys Compd., 434435, 428 (2007).

17. Y. J.V. Ruban, Asian. J. Chem., 20, 4609 (2008).

18. N.I. Alekseev, O.V. Arapov, S.V. Polovtsev, N.A. Charykov, S.G. Izotova and M.G. Potalitsin, Russ. J. Appl. Chem., 78, 2019 (2005).

19. J.R. Yu, N. Grossiord, C.E. Koning and J.C. Loos, Carbon, 45, 618 (2007). 